\title{
PREDICTIVE ANALYSIS FOR LEUKEMIA SURVIVAL USING AI BOTS
}

\author{
G. V. Vaishnavi \\ MCA Scholar,School of CS \& IT, Dept. of MCA,Jain(Deemed-to-be) University, Bangalore
}

Article DOI: https://doi.org/10.36713/epra4327

\begin{abstract}
A Chatbot is an Artificial Intelligence (AI) software that conducts a conversation with users via text-based method without any human intervention at the server side. In this project we implemented only limited features like text-based chatting which is designed only for the people who are dealing with Leukemia. People can ask about anything and everything related to leukemia symptoms and the bot will provide the necessary precautions based on their queries. People can communicate with the chatbot which makes the computers closer to human-level understanding. The Chatbot collects the keywords and will start the conversation by asking queries to the users and provide the suggesting remedies.
\end{abstract}

KEYWORDS- Chatbot, Leukemia, Cancerbot.

\section{INTRODUCTION}

A Chabot is an assistance that individuals communicate with by means of a talk interface. You can pose inquiries utilizing your voice or by composing similarly you would ask an individual. The chatbot will as a rule react in a conversational style, and it might do activities because of your discussion (for model, request something for you). It regularly runs inside a mainstream informing application, for example, Facebook Errand person, Slack, or SMS. It addresses your inquiry, as opposed to guiding you to a site.

Over the last few years, Chatbots have played a very important role as a human-computer interfaces. Chatbots are usually consist of three modules: the user interface, an interpreter, and a knowledge base. Laven describes that chatbot as a program that challenges to imaginary typed conversation, with the aim of at least briefly misleading the human into intellectual while talking to another person. Chatbot is one of the best suitable technologies that industries can rely on, possibly substituting the old models and creating the apps and websites. A Chatbot ("Chatter Robot") is a computer program that converts human dialogues in its usual methods including text or verbal language using artificial intelligence techniques such as Natural Language Processing (NLP), photos and audio-visual processing, and audio analysis. The best exciting feature of the bots is that they learn from the earlier communications and become intelligent and smoother over the time.
Created in 1995, ALICE was the best well-known chatbot of 20th century. Being stimulated by Elizz, it was able to chat with humans more efficiently owing to its experiential purposes. ALICE was also the encouragement for Apple's personal assistant chatbot "Siri". Apart from revolutionary improvements in computer science, ALICE was also the inspiration of many science fiction movies. The evolution in Chatbots came with the launch of Apple's personal assistant Siri. Siri features aa a bright range of user commands, including performance phone actions, scheduling events, checking simple information, reminders, handling device settings, navigating areas, searching the Internet, finding information on entertainment, and is also able to engage with iOS-integrated applications. After the revolution brought by IBM's Watson in terms of natural language processing, others also jumped in to the game. Amazon introduced Alexa \& Google developed dialog agent known as Dialog Flow which offers natural language processing capabilities. Google's agent provides single platform integration with Chabot frameworks of Facebook, Twitter, Skype, Cortana, Alexa, Slack and many more. 2 Chatbots works in two ways- rule based and smart machine based. Rule based chatbots deliver predefined answers from a data base, based on the keywords used for the search. However, smart machine based chatbots inherit its capabilities from Artificial Intelligence and Cognitive Computing and adjust their performance based on the customer communications. The most standard chatbot are various search engines for 


\section{SJIF Impact Factor: 6.260| ISI I.F.Value:1.241| Journal DOI: 10.36713/epra2016 ISSN: 2455-7838(Online) EPRA International Journal of Research and Development (IJRD) Volume: 5 | Issue: 4 | April 2020 - Peer Reviewed Journal}

bots, such as Chatbottle, Botlist and there is a bot for that, for example, helping developers to inform users about the launch of new talkbots. These sites also provide a ranking of bots by various limitations the number of votes, user statistics, platforms, categories (travel, productivity, social interaction, e-commerce, entertainment, news, etc.). They feature more than three and a half thousand bots for Facebook Messenger, Slack, Skype and Kik.

With online life entrance and web network ready to increment joined by propels in common language handling and man-made reasoning, chatbots are relied upon to overwhelm the market. For a designer it is critical to comprehend what the chatbot will offer and what classification the chatbot falls into. This would help pick the calculations or stages and instruments to use to fabricate the bot. It additionally helps the end-clients comprehend what's in store. Here we talk about the sorts of chatbots, the apparatuses and calculations that can be utilized for various kinds of chatbots and give a general engineering that can be followed while building bots. We likewise address the territories where in chatbots are missing and recognize the exploration regions consequently requiring consideration.

Leukemia are caused due to immature growth of blood cells in the bone marrow and leads to improper functioning of body which weakens the immune system.

According to survey of World Health Organization (WHO), depression is becoming one of the most challenging phases for the people who are dealing with health issues. Communication will help the people to improve one's mental stability. To help the people and to solve the queries of the people we are introducing a chatbot. People can communicate with the chatbot which makes the computers closer to humanlevel understanding. The Chabot collects

the keywords and will start the conversation by asking queries to the users and provide the suggesting remedies.

A Chabot is an Artificial Intelligence (AI) software that conducts a conversation with users via text-based method without any human intervention at the server side. In this project we implemented only limited features like text-based chatting which is designed only for the people who are dealing with Leukemia.

\section{LITERATURE REVIEW}

[1] In this paper author proposed a model that overcomes the patients doubt regarding the questions related to cancer. It explained Most cancers chatbot could have a greater great have an effect on the existence of most cancers patients and might assist them to clarify their doubt concerning most cancers. Unlike present chatbots which centered on various domains of healthcare apart from most cancers, this chatbot centered most effective on cancer. Via an interactive session with this most cancers bot, it's far possible for the sufferers to obtain applicable information regarding cancer. This is the first-rate solution for those who are busy with their job schedules. They do not want to attend inside the queue for hours to get an appointment with a health practitioner whenever as a substitute they could chat with the bot. According to survey of World Health Organization (WHO), depression is becoming one of the most challenging phases for the people who are dealing with health issues. Communication will help the people to improve one's mental stability.

[2] In this paper the author proposed a chatbot for breast cancer where the users interacted with the bot to gain information about the breast cancer. The author conducted a study where they tried to analyze the user's emotional intelligence when they communicate with the bot and based on its users got suggestions from the bot and did one-year research to know this can improve the adherence medication rate of the patients.

\section{PROBLEM STATEMENT}

To get doctor's assistance for basic information about illness is not a feasible method because of money constraint, what if the hospital is located in far locations from the patient's home or unavailable of doctor on time due to busy scheduling. Any proposed method that overcomes the above-mentioned scenarios which reduces the bridge between doctor and patient will be a helpful one.

\section{DESIGN}

A Chatbot denotes to a conversation bot. It is a communication pretending computer program. It is all about the discussion with the user. The conversation with a Chatbot is very easy. Its responses to the queries asked by the user. The conversation with the user and the chatbot is very important. The design of chatbot indicates as follows:

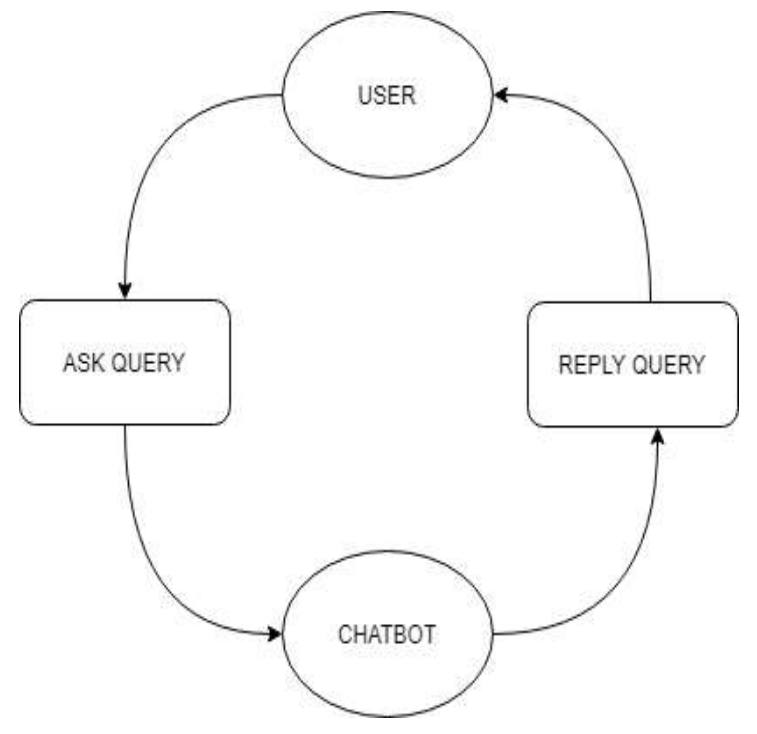

Design of chatbot 


\section{SJIF Impact Factor: 6.260| ISI I.F.Value:1.241| Journal DOI: 10.36713/epra2016 ISSN: 2455-7838(Online) \\ EPRA International Journal of Research and Development (IJRD) \\ Volume: 5 | Issue: 4 | April 2020 \\ - Peer Reviewed Journal}

\section{FLOW CHART}

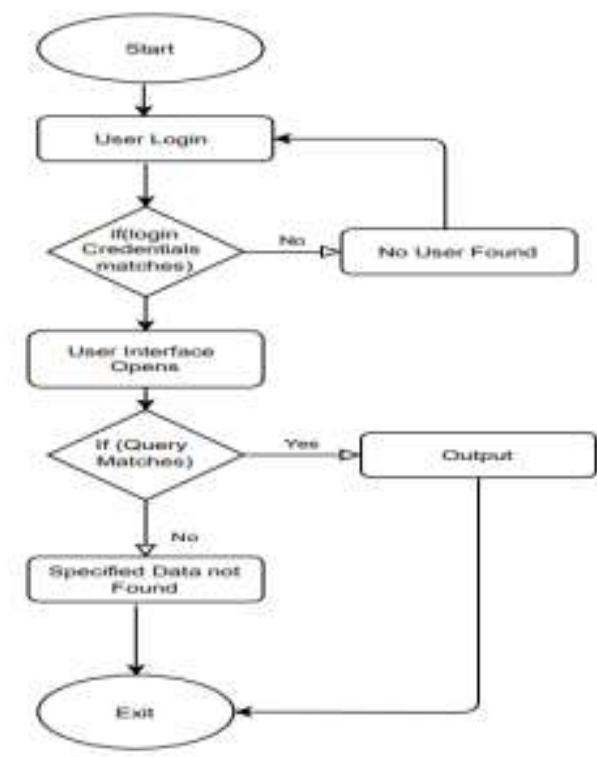

Chabot replies a way it is known to the user. It follows basic English language which will be easy for the user to understand. The conservation between bot and user will be like communicating with each other. The user will login through the interface and it opens the bot where the user information will get stored in the database. The user will give query the bot then it will analyses the user request and send the respond to the user if the query is found the bot will display the output otherwise it will display the specified data is not found.

\section{ARCHITECTURE}

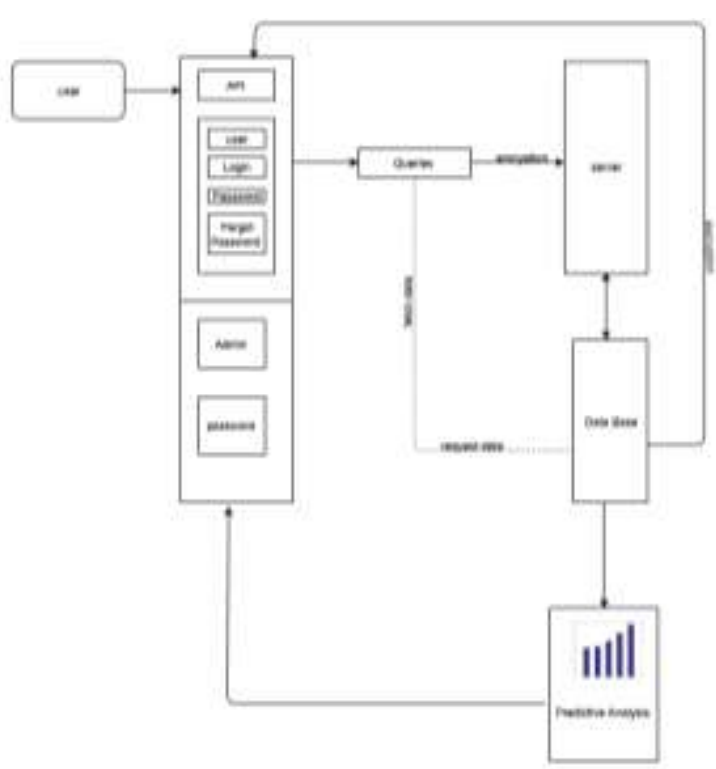

The user will enter the credentials in UI which allows the user to interact with the bot. The user will ask the queries to the bot related to the leukemia the bot will search for the query in the database then displays the corresponding answer based on the user inquiry and from the database it will display the graph based on the user queries.

\section{RESULT AND CONCLUSION}

The main objectives of the project were to develop an algorithm that will be used to identify answers related to user submitted questions. Chatbots are keen associates with man-made consciousness are drastically evolving organizations. There is a wide scope of chatbot building stages that are accessible for different endeavors, for example, internet business, retail, banking, relaxation, travel, human services, etc.

This project explains about the implemented chatbot is a rule based one where the users rely on the list of questions and the corresponding answers. Users can be used to get the required information about Leukemia and that will help the people to understand the symptoms of that particular cancer it will help them guide their closed ones. This Chatbot will save the time of the people if the respected doctor is living far from the place where they live.

\section{FUTURE ENHANCEMENT}

Voice recognition and speech recognition can be added which will help the users to clarify their doubts and can also incorporate speech we must install multiple languages so that the bot can respond in the language the user wants to speak. Can create a better interface where hand gestures can be added for the bot which will make the users feel they are talking to the human instead of bots.

Developing NLO technology for automated calls will also help the users to use the chatbot during critical situations.

\section{REFERENCES}

1. Belfin $R V$, Shobana A J, Megha Manilal, Ashly Ann Mathew, Blessy Babu, A Graph Based Chatbot for Cancer Patients, 2019.

2. Benjamin Chaix, Jean-Emmanuel Bibault, Arthur Pienkowski, Guillaume Delamon, Arthur Guillemassé, Pierre Nectoux, Benoît Brouard, When Chatbots Meet Patients: One-Year Prospective Study of Conversations Between Patients With Breast Cancer and a Chatbot,2019. 